DE DE GRUYTER

OPEN
Journal of Intercultural Management

Vol. 6, No. 2, April 2014, pp. 117-135

DOI 10.2478/joim-2014-0015

\author{
Alba María del Carmen González Vega \\ Universidad Autónoma Metropolitana-Unidad Iztapalapa, México
}

\title{
Caracterización de la participación de la familia en la producción de las empresas en México
}

\section{Characteristics of the participation of the family in the production of companies in Mexico}

\begin{abstract}
In the study of family business there is no concrete data on the number of Mexican companies that are family owned, however in reviewing the Economic Census of the National Institute of Statistics and Geography [INEGI] it can be seen that provided staff busy donot receive a formal economic perception from owners, family and other unpaid worker, so that, by linking the concept of family business and the companies that have reported such staff, this study identifies the behavior of the family business considering the contribution to production by sector and hours worked, as well as by gender, concentration of owners, family and other unpaid workers by sector and firm size (micro, small, medium and large companies). This study provides reference the importance of the family business in Mexico's economic output, setting a mapping behavior from a quantitative approach. It should be noted that this study is part of a doctoral research project in progress over the family business, which is why, it is important to have a reference of the macro context in which the organization is embedded study, presented at the following inquiry.
\end{abstract}

Key-words: Family, company, production sectors.

\section{Introducción}

Dentro de un sistema económico como el capitalista, la figura de la empresa ha sido una expresión concreta de las relaciones productivas de la economía 
mundial. Un tipo de organización que ha sido definida y estudiada ampliamente por diferentes perspectivas, es la empresa familiar, pero fundamentalmente el acercamiento que se ha hecho a esta organización es económico, desde este punto de vista, la empresa es un ente donde las relaciones económicas determinan su origen, su diario actuar, su desarrollo y su muerte; su estudio y comprensión se ha limitado a sus generalidades resaltando básicamente sus relaciones económicas [de la Rosa 2004].

Así el estudio de la empresa familiar en México se ha generado a partir del reconocimiento de la sociedad y el gobierno al identificar el potencial de las mismas en la economía mundial [Ruiz 2003], es por esto que la mayoría de las investigaciones y aportes hechas en torno a ésta, ha girado alrededor de su importancia como actor económico y a sus relaciones productivas, su relación con el empleo, su productividad, su eficiencia y las aportaciones al Producto Interno Bruto (PIB); de esta forma la empresa familiar como aquella forma de organización moderna, su importancia ha radicado, en términos económicos, en el nivel de empleo que proporciona y las aportaciones como agente económico [Gitman \& McDaniel 2007].

Para el estudio de la empresa familiar, no existe una definición única que sea generalmente aceptada, que contenga todas las aristas, que presenten las ópticas económica, jurídica, social, y organizacional. En general se aborda desde diferentes aspectos y problemáticas que procuran explicar la fusión entre la familia, la empresa y la propiedad como una particularidad trascendental y única; consignando un tipo especial de negocio [Ramírez 2008]; bajo esa definición una gran cantidad de empresas en México y en el mundo serían empresas familiares.

Su estudio se ha abocado a caracterizarla a partir del control de la familia en la gestión de la empresa; es decir que, la familia ejerce un control sobre la empresa a partir de su participación en el capital social ${ }^{1}$, dicha participación permite ejercer control - político y económico - sobre la misma; por el lado de la gestión, la inclusión de la familia en puestos de trabajo-operativos o directivos-, o en los órganos de administración (en el caso de gran empresa) para la eficiente toma de decisiones; otro punto es el estudio del fracaso y éxito de la empresa en el proceso de sucesión [Díaz \& Mayett 2011]; así como la continuidad, que hace referencia a la búsqueda de métodos y herramientas que apoyen a la empresa a perpetuarse en el tiempo [Díaz \& Mayett 2011, Belausteguigoitia 2010, Balli 2003, Vélez et al., 2008, Rendón 2006].

Sin embargo, la presente indagación permite tener un acercamiento, de forma general de su comportamiento en la economía mexicana, sin soslayar la

1 El capital social se define como es la cantidad de dinero en efectivo y otros activos propiedad de una empresa. 
complejidad del actuar de los individuos en la dinámica organizacional específica $y$, así poder visualizar algunos patrones de comportamiento permitiendo tener una mirada macro sobre el fenómeno a partir de un enfoque cuantitativo.

\section{Objetivo del estudio}

Caracterizar la participación de la familia en los diferentes sectores empresariales en México en el año 2008 con base en la información recopilada en el Censo Económico 2009 del Instituto Nacional de Geografía y Estadística INEGI.

Preguntas específicas que interesa responder con el análisis.

- ¿Qué proporción de las empresas en México son empresas familiares?

- ¿Qué proporción de las empresas en México no son empresas familiares?

- ¿Qué sector económico tiene la mayor concentración de familia?

- ¿Qué tipo de empresa tiene una mayor concentración de familia?

- En la familia, ¿qué genero tiene una mayor concentración en la empresa familiar? ¿cuál es su distribución por sector productivo?

\section{Método}

Los datos que se utilizaron en el presente estudio se basan en el Censo Económico de 2009 del Instituto Nacional de Geografía y Estadística (INEGI) para el caso específico de las empresas familiares caracterizadas por:

- Concentración de propietarios, familiares y otros trabajadores no remunerados por Entidad federativa por sector de actividad

- Concentración de propietarios, familiares y otros trabajadores no remunerados por tamaño de las unidades económica por sector de actividad.

- Personal ocupado remunerado.

- Personal ocupado (propietarios, familiares y otros trabajadores no remunerados)

- Horas trabajadas.

- Género.

La finalidad principal es caracterizar la participación de la familia en los diferentes sectores empresariales en México en el año 2008 con base en la información recopilada en el Censo Económico 2009 del Instituto Nacional de Geografía y Estadística INEGI y considerando la estratificación (ver tabla 1). 
Tabla 1 Estratificación de las empresas en México

\begin{tabular}{|c|c|c|c|c|}
\hline \multicolumn{5}{|c|}{ Estratificación } \\
\hline Tamaño & Sector & $\begin{array}{l}\text { Rango de } \\
\text { número de } \\
\text { trabajadores }\end{array}$ & $\begin{array}{l}\text { Rango de monto } \\
\text { de } \\
\text { ventas anuales } \\
\text { (mdp) }\end{array}$ & $\begin{array}{l}\text { Tope máximo } \\
\text { combinado* }\end{array}$ \\
\hline Micro & Todas & Hasta 10 & Hasta $\$ 4$ & 4.6 \\
\hline \multirow{2}{*}{ Pequeña } & Comercio & $\begin{array}{l}\text { Desde } 11 \text { hasta } \\
30\end{array}$ & $\begin{array}{l}\text { Desde } \$ 4.01 \text { hasta } \\
\$ 100\end{array}$ & 93 \\
\hline & Industria y Servicios & \begin{tabular}{|l} 
Desde 11 hasta \\
50
\end{tabular} & $\begin{array}{l}\text { Desde } \$ 4.01 \text { hasta } \\
\$ 100\end{array}$ & 95 \\
\hline \multirow{3}{*}{ Mediana } & Comercio & $\begin{array}{l}\text { Desde } 31 \text { hasta } \\
100\end{array}$ & \multirow{2}{*}{$\begin{array}{l}\text { Desde } \$ 100.01 \\
\text { hasta } \\
\$ 250\end{array}$} & \multirow{2}{*}{235} \\
\hline & Servicios & $\begin{array}{l}\text { Desde } 51 \text { hasta } \\
100\end{array}$ & & \\
\hline & Industria & $\begin{array}{l}\text { Desde } 51 \text { hasta } \\
250\end{array}$ & $\begin{array}{l}\text { Desde } \$ 100.01 \text { has } \\
\text { ta } \\
\$ 250\end{array}$ & 250 \\
\hline
\end{tabular}

*El tamaño de la empresa se determinará a partir del puntaje obtenido conforme a la siguiente fórmula: Puntaje de la empresa = (Número de trabajadores) $\times 10 \%+$ (Monto de Ventas Anuales) $\times 90 \%$, el cual debe ser igual o menor al Tope Máximo Combinado de su categoría.

Fuente: Ley para el Desarrollo de la Competitividad de la Micro, Pequeña y Mediana Empresa (DOF, 30/06/2009)

Para la categorización de la información se utilizó la clasificación de los sectores económicos propuesta por el INEGI en el Sistema de Clasificación Industrial de América del Norte ${ }^{2}$ [SCIAN 2007] (ver tabla 2) con la finalidad de identificar la forma en cómo se catalogan los sectores productivos en México y que tipo de unidades económicas integran a estos.

Tabla 2 Clasificación de los sectores en México

$\begin{aligned} & \text { Característica general de Sector } \\
& \text { los sectores }\end{aligned}$
\begin{tabular}{|l|l|l|}
$\begin{array}{l}\text { Explotación de recursos } \\
\text { naturales }\end{array}$ & 11 & $\begin{array}{l}\text { Agricultura, cría y explotación de animales, aprovechamien- } \\
\text { to forestal, pesca y caza }\end{array}$ \\
\hline Transformación de bienes & 21 & Minería \\
\hline Transformación de bienes & 22 & $\begin{array}{l}\text { Generación, transmisión y distribución de energía eléctrica, } \\
\text { suministro de agua y de gas por ductos al consumidor final }\end{array}$ \\
\hline Transformación de bienes & 23 & Construcción \\
\hline Transformación de bienes & $31-33$ & Industrias manufactureras \\
\hline Distribución de bienes & 43 & Comercio al por mayor \\
\hline
\end{tabular}

2 Ver anexo 1 


\begin{tabular}{|c|c|c|}
\hline $\begin{array}{l}\text { Característica general de } \\
\text { los sectores }\end{array}$ & Sector & Criterios de orden \\
\hline Distribución de bienes & 46 & Comercio al por menor \\
\hline Distribución de bienes & $48-49$ & Transportes, correos y almacenamiento \\
\hline $\begin{array}{l}\text { Operaciones con infor- } \\
\text { mación }\end{array}$ & 51 & Información en medios masivos \\
\hline Operaciones con activos & 52 & Servicios financieros y de seguros \\
\hline Operaciones con activos & 53 & $\begin{array}{l}\text { Servicios inmobiliarios y de alquiler de bienes muebles e } \\
\text { intangibles }\end{array}$ \\
\hline $\begin{array}{l}\text { Servicios cuyo insumo } \\
\text { principal es el conocimien- } \\
\text { to y la experiencia del } \\
\text { personal }\end{array}$ & 54 & Servicios profesionales, científicos y técnicos \\
\hline $\begin{array}{l}\text { Servicios cuyo insumo } \\
\text { principal es el conocimien- } \\
\text { to y la experiencia del } \\
\text { personal }\end{array}$ & 55 & Corporativos \\
\hline $\begin{array}{l}\text { Servicios cuyo insumo } \\
\text { principal es el conocimien- } \\
\text { to y la experiencia del } \\
\text { personal }\end{array}$ & 56 & $\begin{array}{l}\text { Servicios de apoyo a los negocios y manejo de desechos y } \\
\text { servicios de remediación }\end{array}$ \\
\hline $\begin{array}{l}\text { Servicios cuyo insumo } \\
\text { principal es el conocimien- } \\
\text { to y la experiencia del } \\
\text { personal }\end{array}$ & 61 & Servicios educativos \\
\hline $\begin{array}{l}\text { Servicios cuyo insumo } \\
\text { principal es el conocimien- } \\
\text { to y la experiencia del } \\
\text { personal }\end{array}$ & 62 & Servicios de salud y de asistencia social \\
\hline $\begin{array}{l}\text { Servicios relacionados con } \\
\text { la recreación }\end{array}$ & 71 & $\begin{array}{l}\text { Servicios de esparcimiento culturales y deportivos, y otros } \\
\text { servicios reacreativos }\end{array}$ \\
\hline $\begin{array}{l}\text { Servicios relacionados con } \\
\text { la recreación }\end{array}$ & 72 & $\begin{array}{l}\text { Servicios de alojamiento temporal y de preparación de ali- } \\
\text { mentos y bebidas }\end{array}$ \\
\hline Servicios residuales & 81 & Otros servicios excepto actividades gubernamentales \\
\hline $\begin{array}{l}\text { Actividades gubernamen- } \\
\text { tales }\end{array}$ & 93 & $\begin{array}{l}\text { Actividades legislativas, gubernamentales, de impartición de } \\
\text { justicia y de organismos internacionales y extraterritoriales }\end{array}$ \\
\hline
\end{tabular}

Fuente. INEGI [2007]

En cuanto a la caracterización de la familia en las empresas mexicanas, se toma como referencia el siguiente concepto: Para Díaz \& Mayett [2011] una empresa se puede considerar familiar si se dan las siguientes circunstancias:

- Una familia posee una participación significativa de su capital social, normalmente la mayoría.

- Dicha participación permite a la familia ejercer control-político y económicosobre la empresa. 
- La familia participa en la gestión de la empresa de manera activa, mediante el trabajo de la misma de alguno o algunos de sus miembros, generalmente en puestos directivos o en el órgano de administración.

- El deseo de los propietarios de que la empresa continúe en manos de la propia familia mediante la incorporación de la siguiente generación familiar a la propiedad y, en su caso, a la gestión y/o al gobierno de la misma.

Para el presente caso, se identificará como empresa familiar aquella que tenga dos o más miembros participando en la empresa. Para su tipificación en el Censo Económico se retoma al personal no remunerado (propietarios, familiares y otros trabajadores no remunerados) siendo estos "las personas que trabajaron para la unidad económica, cubriendo como mínimo una tercera parte de la jornada laboral de la misma sin recibir un sueldo o salario" [INEGI, 2009]. Se consideran aquí a los propietarios, familiares de éstos, socios activos, prestadores de servicio social, personas de programas de empleo, etcétera. Excluye: a quienes prestaron sus servicios profesionales o técnicos y cobraron honorarios por ello; pensionados o jubilados, y al personal suministrado por otra razón social" [INEGI, 2007].

Para poder localizar y referenciar la distribución geográfica por sector y tipo de empresa se hace una división de las entidades federales en cinco regiones con base en el Diario Oficial de la Federación (ver mapa 1):

1. Zona Noroeste: Baja California, Baja California Sur, Chihuahua, Sinaloa y Sonora.

2. Zona Noreste: Coahuila, Durango, Nuevo León, San Luis Potosí y Tamaulipas.

3. Zona Occidente: Aguascalientes, Colima, Guanajuato, Jalisco, Michoacán, Nayarit, Querétaro y Zacatecas.

4. Zona Centro: Distrito Federal, Estado de México, Guerrero, Hidalgo, Morelos, Puebla y Tlaxcala.

5. Zona Sureste: Campeche, Chiapas, Oaxaca, Quintana Roo, Tabasco, Veracruz y Yucatán.

Para poder hacer una clasificación de la concentración de los propietarios, familiares y otros trabajadores no remunerados, se estableció una tipificación (ver tabla 3), esto con la finalidad de poder referenciarlo al espacio geográfico.

Tabla 3 Tipificación por grado de concentración de los propietarios, familiares y otros trabajadores no remunerados

\begin{tabular}{|l|l|}
\hline Tipo de concentración & Rango \\
\hline Baja & Menor de $26 \%$ \\
\hline Media & $\geq 26 \% \mathrm{y} \leq 36 \%$ \\
\hline Alta & $\geq 37 \% \mathrm{y} \leq 50 \%$ \\
\hline Sobre saliente & Mayor del $51 \%$ \\
\hline
\end{tabular}

Fuente: elaboración propia 
${ }^{3}$ Mapa 1 División de la República Mexicana en regiones

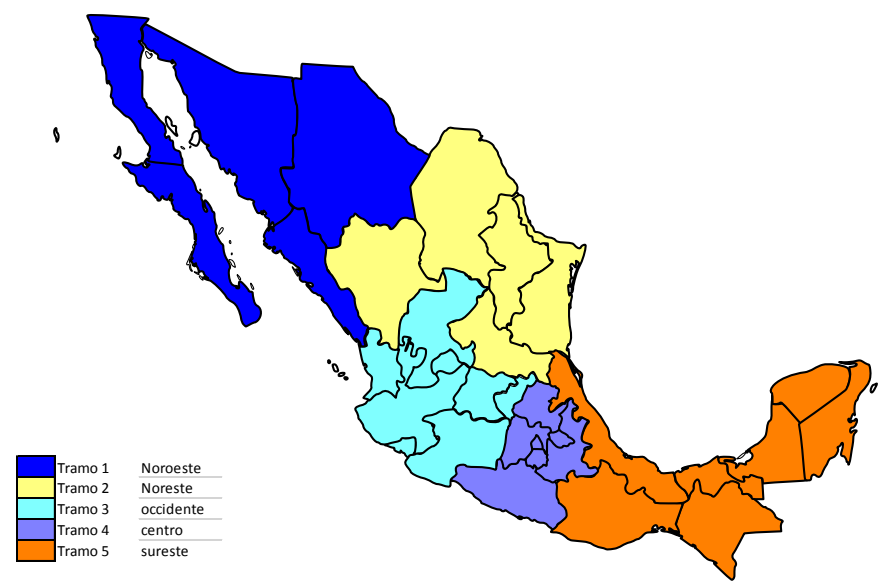

Fuente: DOF 10/01/2013

Comportamiento general de la empresa familiar en México: concentración y distribución geográfica por regiones, sector y tamaño de empresa.

Contexto general de la empresa mexicana

Dentro de un sistema económico como el capitalista, la figura de la empresa ha sido una expresión concreta de las relaciones productivas de la economía mundial. Un tipo de organización que ha sido definida y estudiada ampliamente por diferentes perspectivas, es la pequeña empresa, pero fundamentalmente el acercamiento que se ha hecho a esta organización es económico, desde este punto de vista, la empresa es un ente donde las relaciones económicas determinan su origen, su diario actuar, su desarrollo y su muerte; su estudio y comprensión se ha limitado a sus generalidades resaltando básicamente sus relaciones económicas [de la Rosa, 2004].

Así el estudio de la empresa familiar en México se ha generado a partir del reconocimiento de la sociedad y el gobierno al identificar el potencial de las mismas en la economía mundial [Ruiz, 1995], es por esto que la mayoría de las investigaciones y aportes hechas en torno a ésta, ha girado alrededor de su importancia como actor económico y a sus relaciones productivas, su relación con el empleo, su productividad, su eficiencia y las aportaciones al Producto Interno Bruto (PIB); de esta forma la empresa familiar como aquella forma de

3 La división de la República Mexicana en regiones que se utiliza, hace referencia únicamente a visualizar el espacio geográfico por grupo de entidades federativas. 
organización moderna, su importancia ha radicado, en términos económicos, en el nivel de empleo que proporciona y las aportaciones como agente económico [Gitman y McDaniel, 2001]. De esta forma para poder tener una idea de cómo se comporta la economía mexicana primero se presenta, de forma general, la concentración de empresas por sector de actividad (Ver gráfica 1) y por personal ocupado (ver gráfica 2).

Gráfica 1 Concentración de las empresas por sector de actividad

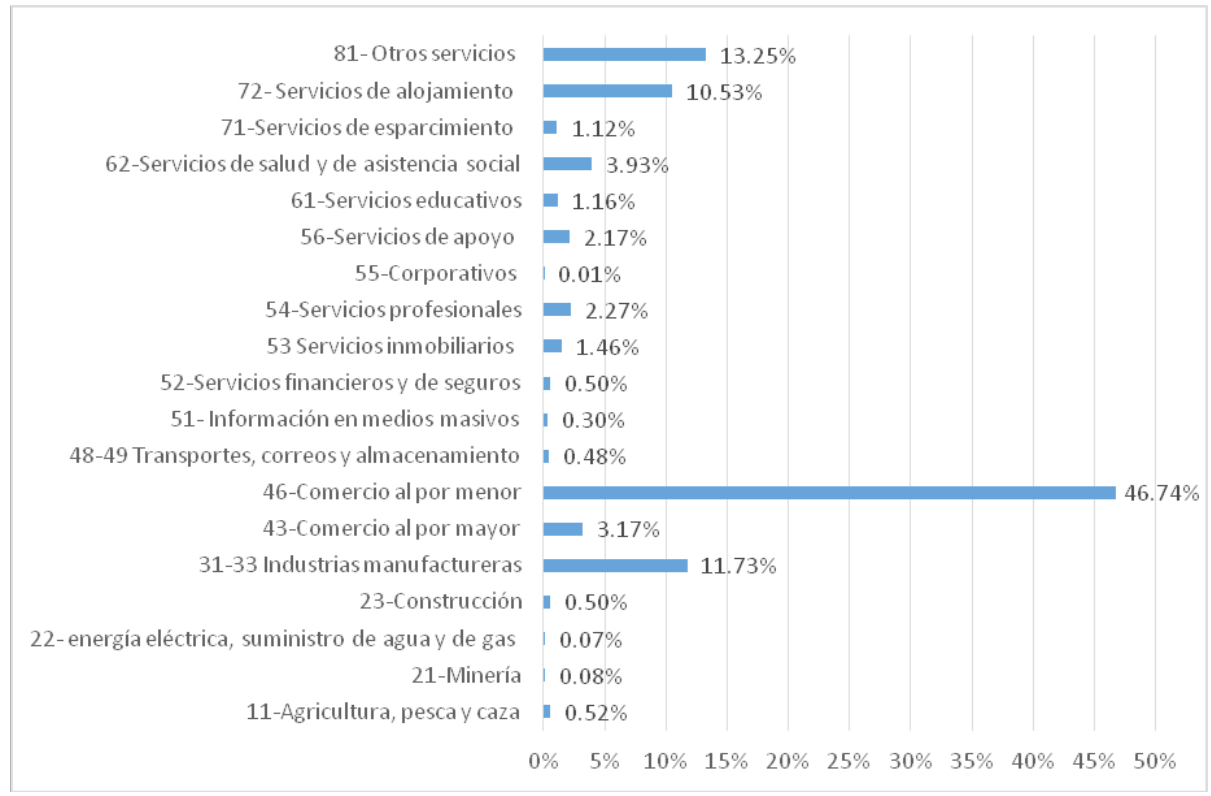

Fuente: Censo Económico 2009

Gráfica 2 Personal ocupado total por sector de actividad

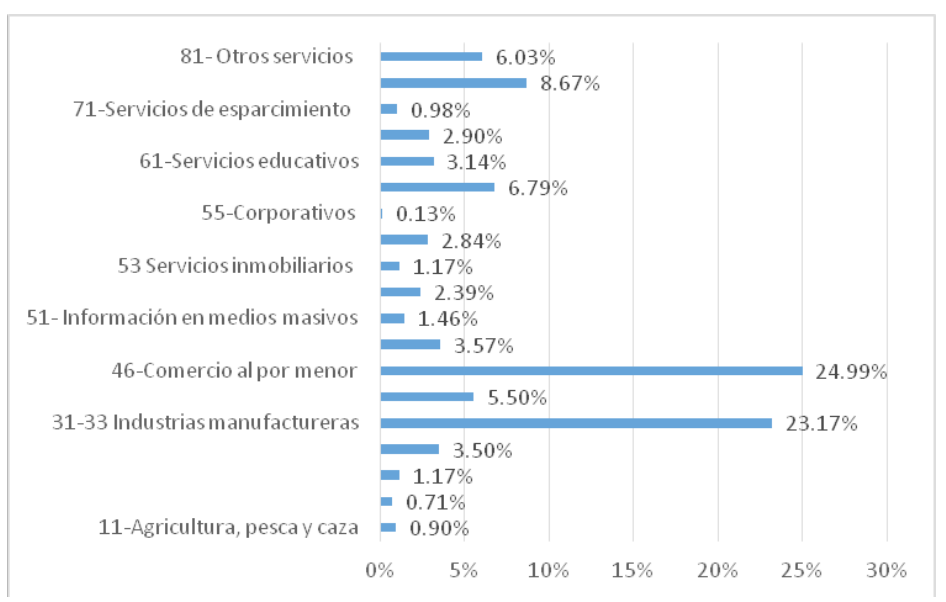

Fuente: Censo Económico 2009 
Lo que se observa en las gráficas 1 y 2 determina que México y su actividad económica está, básicamente, basada en el comercio al por menor (sector 46), el cual concentra de personal ocupado total el $24.99 \%$, siendo estos los porcentajes más altos.

En cuanto al tamaño, el comportamiento de la micro, pequeña, mediana y gran empresa es la siguiente:

Tabla 4 Concentración por tamaño de empresa

\begin{tabular}{|lllll|}
\hline $\begin{array}{l}\text { DENOMI- } \\
\text { NACIÓN }\end{array}$ & $\begin{array}{l}\text { UNIDADES } \\
\text { ECONÓMI- } \\
\text { CAS }\end{array}$ & PORCENTAJE & $\begin{array}{l}\text { PERSONAL } \\
\text { OCUPADO } \\
\text { TOTAL }\end{array}$ \\
\hline MICRO & $3,536,178$ & $94.96 \%$ & $8,414,444$ & $41.83 \%$ \\
\hline PEQUENA & 125,280 & $3.36 \%$ & $2,117,602$ & $10.53 \%$ \\
\hline MEDIANA & 43,356 & $1.16 \%$ & $2,273,951$ & $11.30 \%$ \\
\hline GRANDE & 19,205 & $0.52 \%$ & $7,310,837$ & $36.34 \%$ \\
\hline TOTAL & $3,724,019$ & $100.00 \%$ & $20,116,834$ & $100.00 \%$ \\
\hline
\end{tabular}

Fuente: elaboración propia con base en el Censo Económico 2009

Como se observa México tiene una concentración de micro empresas con el $94.96 \%$ y el $5.04 \%$ distribuido en la pequeña, mediana y gran empresa; sin embargo el personal ocupado, aunque sigue siendo alto en la micro empresa, con el $41.83 \%$, en el caso de la gran empresa tiene una concentración del 36.34\%; porcentaje alto con respecto a la proporción de micros y grandes empresas. Con base en los datos obtenidos por el Censo Económico 2009, México y su economía principalmente, se sustenta en el comercio al por menor y en las micro empresas.

\section{La empresa familiar en México}

El presente apartado configura la forma en la que se comporta la empresa familiar en México, en qué región y sector se concentra, y cuál es la relación que guarda con el tamaño de la empresa.

Lo primero que se encontró a nivel general y según la definición de empresa familiar, la mayor parte de las empresas en México son familiares, desde las micro hasta las grandes, estadísticamente y como punto de referencia en el Censo Económico 2009 se observó que todos los sectores y las micro, pequeñas, medianas y grandes empresas tienen propietarios, familiares y otros trabajadores no remunerados. Este punto es importante porque determina que se necesita una definición menos ambigua de la empresa familiar y que no sólo hable de la fusión entre la familia, la empresa y la propiedad como una particularidad trascendental y única [Díaz \& Mayett, 2011, Belausteguigoitia, 2010, Ramírez, 2008, De la Rosa, Lozano, \& Ramírez, 2009]. 
Los datos apuntan que el conjunto de empresas por entidad federativa tiene una concentración que va desde el $17.63 \%$ con Nuevo León, siendo éste el más bajo a nivel nacional en comparación con Guerrero que tiene el $63.12 \%$ de propietarios, familiares y otros trabajadores no remunerados (ver gráfica 3 ).

Gráfica 3 Personal dependiente de la razón social

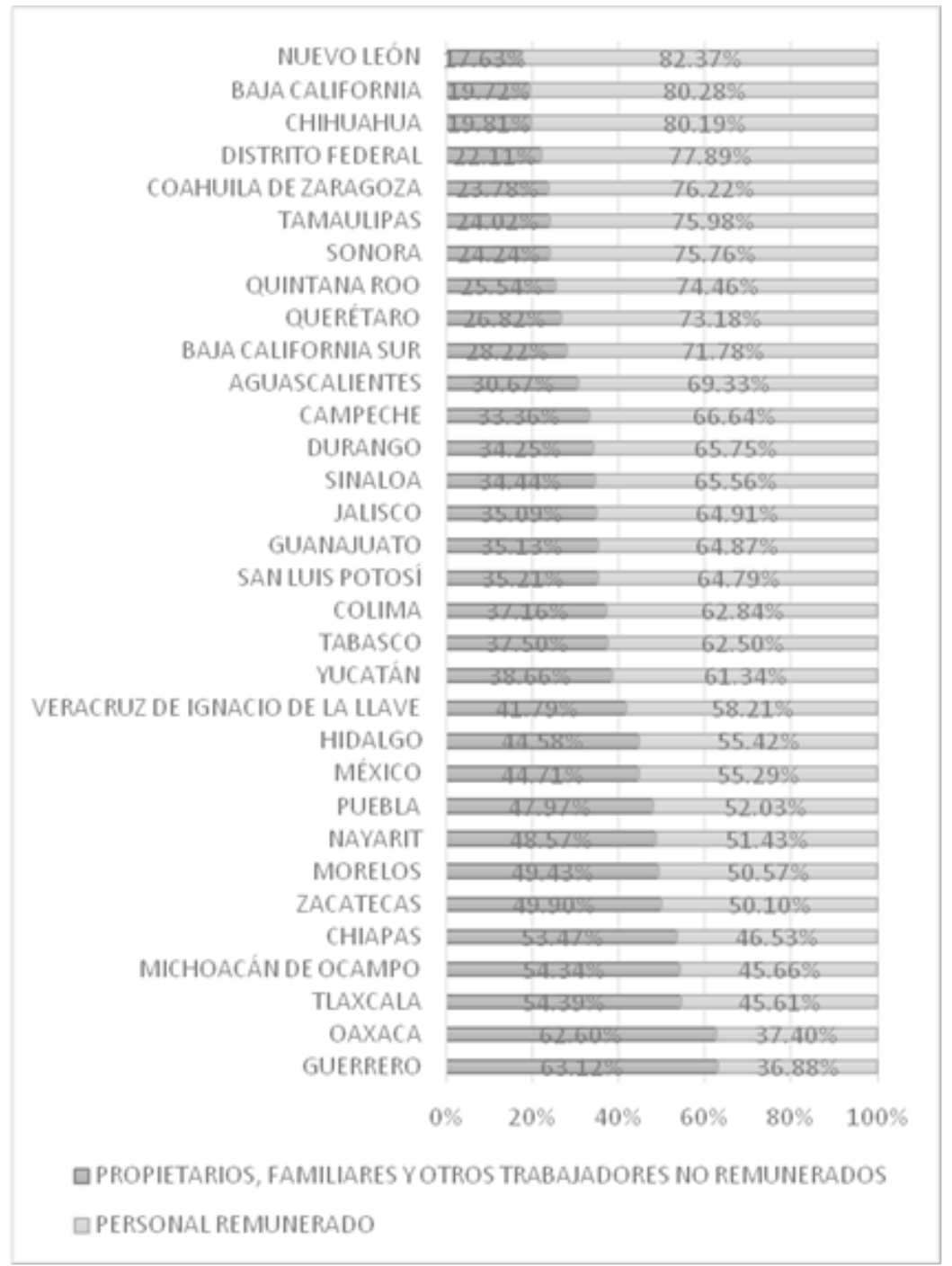

Fuente: elaboración propia 
Con base en la gráfica 3 se exploró la relación de la concentración de los propietarios, familiares y otros trabajadores no remunerados con el espacio geográfico y se encontró lo siguiente:

Mapa 2 Concentración de propietarios, familiares y otros trabajadores no remunerados por entidad federativa

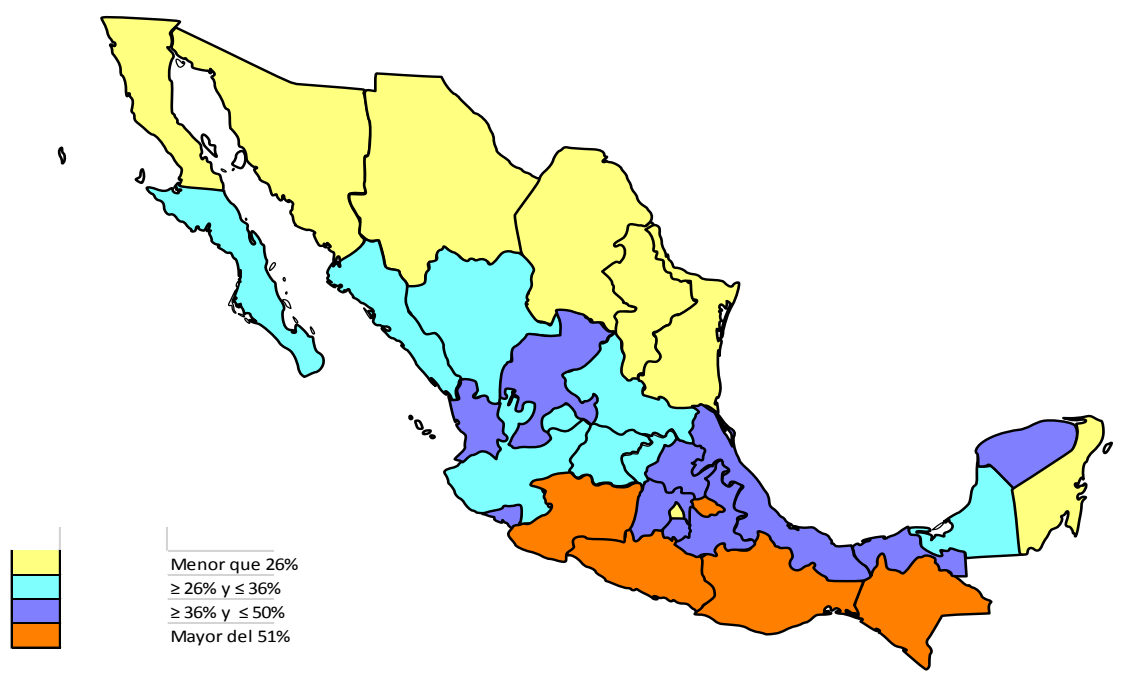

Fuente: elaboración propia con base en la gráfica 3

Como se observa hay dos zonas claramente contrastantes, la zona que se encuentra en color amarillo cerca de la frontera con Estados Unidos de Norteamérica (excepto Quintana Roo y el Distrito Federal que se encuentran alejados) donde la concentración de propietarios, familiares y otros trabajadores no remunerados es baja con una concentración menor al $26 \%$ y aquella en color naranja que agrupa a las entidades federativas que tienen más del $51 \%$. En el caso de la zona fronteriza marcada de amarillo, puede deberse la baja concentración debido a que la mayoría de las empresas se dedica a la manufactura [Mendoza, 2006] cuyo sector, a nivel nacional, presenta una concentración de propietarios, familiares y otro trabajadores no remunerados de $17 \%$.

En contraste con la zona naranja donde los sectores económicos que presentan una mayor concentración de familia son el sector 11 agricultura, cría y explotación de animales, aprovechamiento forestal, pesca y caza, sector 46 comercio al por menor, sector 53 servicios inmobiliarios y de alquiler de bienes muebles e intangibles, sector 62 servicios de salud y de asistencia social, 
sector 71 servicios de esparcimiento culturales y deportivos, y otros servicios recreativos, sector 72 servicios de alojamiento temporal y de preparación de alimentos y bebidas, y sector 81 otros servicios excepto actividades gubernamentales, actividades productivas relacionadas con la región sureste.

Regiones y familia

Al definir los datos en las Regiones establecidas se observan dos tendencias; la primera, es que las regiones noroeste y noreste tienen una tendencia marcada hacia la concentración baja de propietarios, familiares y otros trabajadores no remunerados, esto con relación a la dinámica económica del país, puede referirse a que la frontera norte presenta índices de crecimiento superiores y un desarrollo económico más alto en comparación con las otras regiones [Aguilar, 2007] (Ver mapa 3).

Esto puede llegar a tener una relación con el Producto Interno Bruto y con la concentración de propietarios, familiares y otros trabajadores no remunerados. El segundo punto, es que conforme se baja en el territorio nacional, rumbo al sureste, la concentración porcentual de la familia crece. Los datos señalan una estructura precisa del comportamiento de las empresas familiares y de la economía nacional, en el cual identifica las diferencias marcadas en los índices de crecimiento y desarrollo económico entre el norte y el sur [Aguilar, 2007].

Mapa 3 Concentración de propietarios, familiares y otros trabajadores no remunerados por regiones

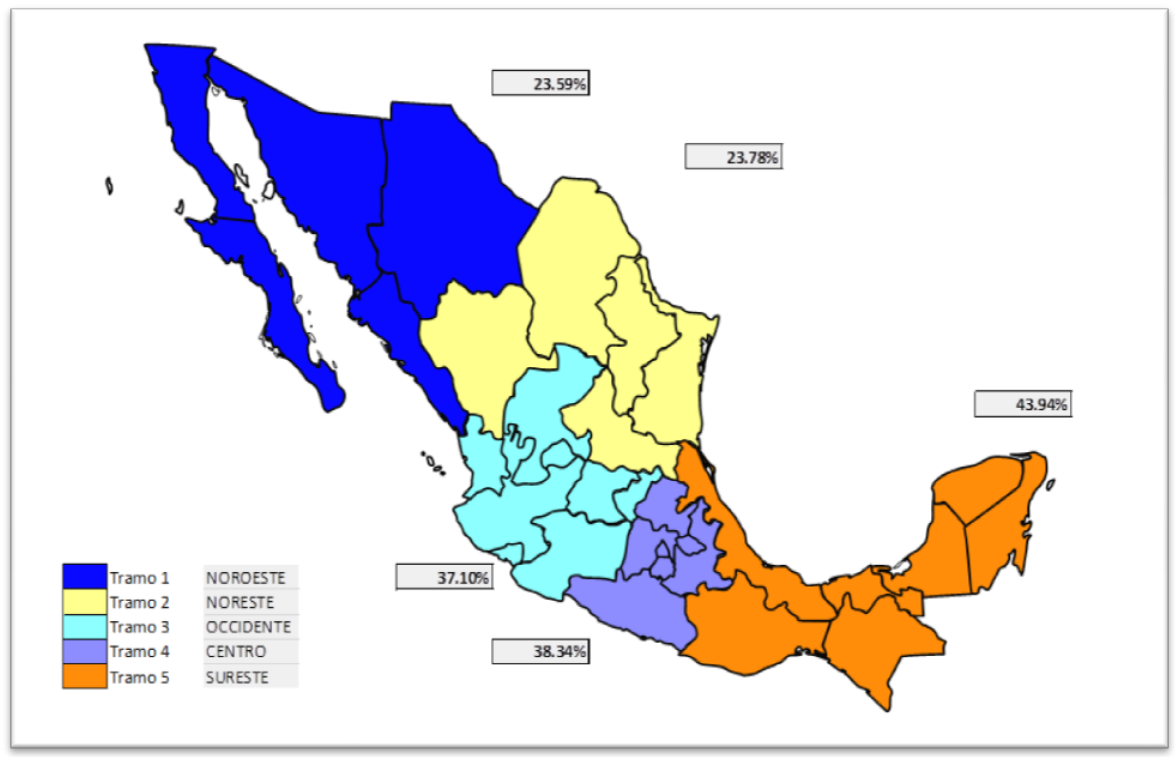

Fuente: Elaboración propia con base en los datos obtenido en el Censo Económico 2009 


\section{Sectores y familia}

En cuanto al comportamiento de los sectores de actividad y la concentración de propietarios, familiares y otros trabajadores no remunerados se observa que los sectores 11, 46, 62 y 81 presentan una concentración de propietarios, familiares y otros trabajadores no remunerados de más del $50 \%$. En relación con la población de empresas, las cuales, al agruparlas representan el $64.44 \%$; es decir esto puede ser indicativo del comportamiento y estructura de las familias empresarias, ya que se desconocen datos concretos [De la Rosa \& Lozano, 2011] (Ver tabla 5).

Tabla 5 Concentración de los propietarios, familiares y otros trabajadores no remunerados por sector de actividad

\begin{tabular}{|l|l|l|l|l|}
\hline Sector & Empresas & porcentaje & $\begin{array}{l}\text { Personal re- } \\
\text { munerado }\end{array}$ & $\begin{array}{l}\text { Propietar- } \\
\text { ios, famili- } \\
\text { ares y otros } \\
\text { trabajadores } \\
\text { no remu- } \\
\text { nerados }\end{array}$ \\
\hline NACIONAL & $3,724,019$ & $100 \%$ & $65.52 \%$ & $34.48 \%$ \\
\hline $\begin{array}{l}11-\text { Agricultura, pesca y } \\
\text { caza }\end{array}$ & 19,443 & $0.52 \%$ & $31.39 \%$ & $68.61 \%$ \\
\hline $\begin{array}{l}46-\text { Comercio al por menor } \\
\text { 81- Otros servicios }\end{array}$ & $1,740,522$ & $46.74 \%$ & $37.81 \%$ & $62.19 \%$ \\
\hline $\begin{array}{l}\text { 62-Servicios de salud y de } \\
\text { asistencia social }\end{array}$ & 493,337 & $13.25 \%$ & $39.09 \%$ & $60.91 \%$ \\
\hline $\begin{array}{l}72-\text { Servicios de aloja- } \\
\text { miento }\end{array}$ & 392,242 & $10.53 \%$ & $48.60 \%$ & $51.40 \%$ \\
\hline 53 Servicios inmobiliarios & 54,188 & $1.46 \%$ & $53.34 \%$ & $46.66 \%$ \\
\hline $\begin{array}{l}71-\text { Servicios de espar- } \\
\text { cimiento }\end{array}$ & 41,821 & $1.12 \%$ & $56.02 \%$ & $43.98 \%$ \\
\hline $\begin{array}{l}54-\text { Servicios profesion- } \\
\text { ales }\end{array}$ & 84,695 & $2.27 \%$ & $57.58 \%$ & $42.42 \%$ \\
\hline $\begin{array}{l}\text { 31-33 Industrias manu- } \\
\text { factureras }\end{array}$ & 436,851 & $11.73 \%$ & $72.41 \%$ & $27.59 \%$ \\
\hline 43-Comercio al por mayor & 118,028 & $3.17 \%$ & $82.05 \%$ & $17.95 \%$ \\
\hline $\begin{array}{l}48-49 \text { Transportes, corre- } \\
\text { os yalmacenamiento }\end{array}$ & 17,705 & $0.48 \%$ & $82.51 \%$ & $17.49 \%$ \\
\hline 61-Servicios educativos & 43,286 & $1.16 \%$ & $88.08 \%$ & $11.92 \%$ \\
\hline 56-Servicios de apoyo & 80,922 & $2.17 \%$ & $88.08 \%$ & $11.92 \%$ \\
\hline 21-Minería & 2,957 & $0.08 \%$ & $90.96 \%$ & $9.04 \%$ \\
\hline $\begin{array}{l}\text { 51- Información en me- } \\
\text { dios masivos }\end{array}$ & 11,354 & $0.30 \%$ & $92.41 \%$ & $7.59 \%$ \\
\hline $\begin{array}{l}52-\text { Servicios financieros y } \\
\text { de seguros }\end{array}$ & 18,706 & $0.50 \%$ & $93.17 \%$ & $6.83 \%$ \\
\hline 23-Construcción & 18,637 & $0.50 \%$ & $95.15 \%$ & $4.85 \%$ \\
\hline
\end{tabular}




\begin{tabular}{|l|l|l|l|l|}
\hline $\begin{array}{l}\text { 22- energía eléctrica, } \\
\text { suministro de agua y de } \\
\text { gas }\end{array}$ & 2,589 & $0.07 \%$ & $98.18 \%$ & $1.82 \%$ \\
\hline 55 -Corporativos & 204 & $0.01 \%$ & $99.98 \%$ & $0.02 \%$ \\
\hline
\end{tabular}

Fuente: Elaboración propia con base en los datos obtenido en el Censo Económico 2009

Por otro lado, los sectores marcados de color amarillo representan a los de menor concentración de propietarios, familiares y otros trabajadores no remunerados, siendo el más alto el sector 31-33 que es de manufacturas y el menor el sector 55 de corporativos (outsourcing) el cual es el que se manifiesta con menor concentración de propietarios, familiares y otros trabajadores no remunerados a nivel nacional.

Tamaño de la empresa y la familia

En cuanto al tamaño, se encontró lo siguiente que las microempresas tienen una mayor concentración de propietarios, familiares y otros trabajadores no remunerados representada por el $67.47 \%$ y gráficamente se observa que existe una relación entre el tamaño y la proporción de la familia en la empresa; es decir, entre menor sea el tamaño de la empresa mayor es el concentración de propietarios, familiares y otros trabajadores no remunerados.

${ }^{4}$ Gráfica 4 Concentración de propietarios, familiares y otros trabajadores no remunerados por tamaño de empresa

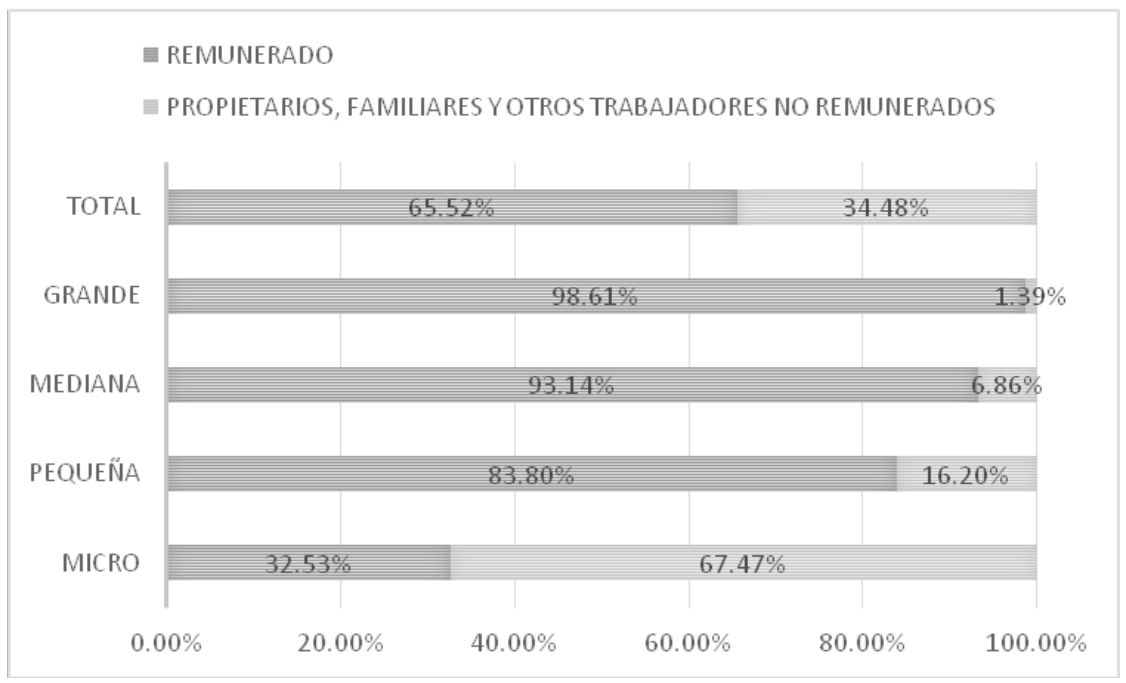

Fuente: Elaboración propia con base en los datos obtenido en el Censo Económico 2009

4 Mintzberg [2002] la normalización se refiere a programar y especificar formalmente los proceso de trabajo; esto es, vía escrita. 
Para Mintzberg [2002] existen dos hipótesis en cuanto al tamaño de la organización:

Hipótesis 1: cuanto mayor sea el uso de la normalización para la coordinación, mayor será la unidad de trabajo.

Hipótesis 2: cuanto más se dependa de la adaptación mutua, menor será el tamaño de la unidad de trabajo.

Esto en relación con la empresa familiar determina dos puntos: el primero, que en aquellas microempresas, no sólo la familia dirige sino opera, es decir la familia aporta horas de trabajo y la coordinación del trabajo se ve establecida por la adaptación mutua y la supervisión directa; en el caso de la gran empresa, en la mayoría de los casos, la familia dirige pero ya no opera o en otro caso, ya sólo tiene acciones [Belausteguigoitia, 2010]; es decir su función preponderante se limita a la toma de decisiones estratégicas; esto puede dar una explicación a este comportamiento; sin embargo, estas empresas no dejan de ser familiares, solamente cambia la posición que toma la familia en cuanto a la operatividad de la organización.

Género, familia y empresa

La inserción de la mujer en las empresas ha traído cambios sustanciales en diversas dimensiones de la vida cotidiana, y posiblemente el impacto más significativo sea sobre la familia. En el caso de la empresa familiar reformula los diferentes roles que tiene que llevar a cabo basadas en las conductas estereotipadas por la cultura sustentadas en creencias y atribuciones sociales [INMUJERES, 2007]; como aquellas que determina su actuar; es decir la mujer cuida de los hijos y se encarga de las labores de la casa; sin embargo, la mujer se ha insertado precipitadamente en la actividades económicas; este es el caso de las empresas familiares.

En la gráfica 5 se observa la concentración de la mujer y los hombres en la actividad productiva de México para el caso de la concentración de propietarios, familiares y otros trabajadores no remunerados, como se observa las mujeres que tienen una mayor incidencia en los sectores 46 comercio al por menor, el 61 de servicios educativos y el 72 de servicios de alojamiento temporal y de preparación de alimentos y bebidas con una concentración de propietarios, familiares y otros trabajadores no remunerados mayor al 50\%; en el otro rango que va del 30 al $40 \%$ se encuentran aquellos sectores dedicados a las actividades terciarias; esto puede indicar un inserción paulatina creciente de la mujer en todos los sectores de actividad productiva rompiendo estereotipos [Barbeito, Nieto de Almeida, \& Pereira, 2011] [INMUJERES, 2007] , como lo menciona Blanco [1999]: 
"la fuerza de trabajo femenina se ha incorporado mayoritariamente al sector de los servicios (incluyendo el comercio), aunque en el caso de México cabe destacar la importante presencia de la industria maquiladora como fuente de trabajo para las mujeres; de tal suerte que, en conjunto, se habla del fenómeno de la feminización de los mercados de trabajo" (p. 29)

Gráfica 5 Concentración de Propietarios, familiares y otros trabajadores no remunerados por género

Propietarios, familiares y personal no remunerado por género

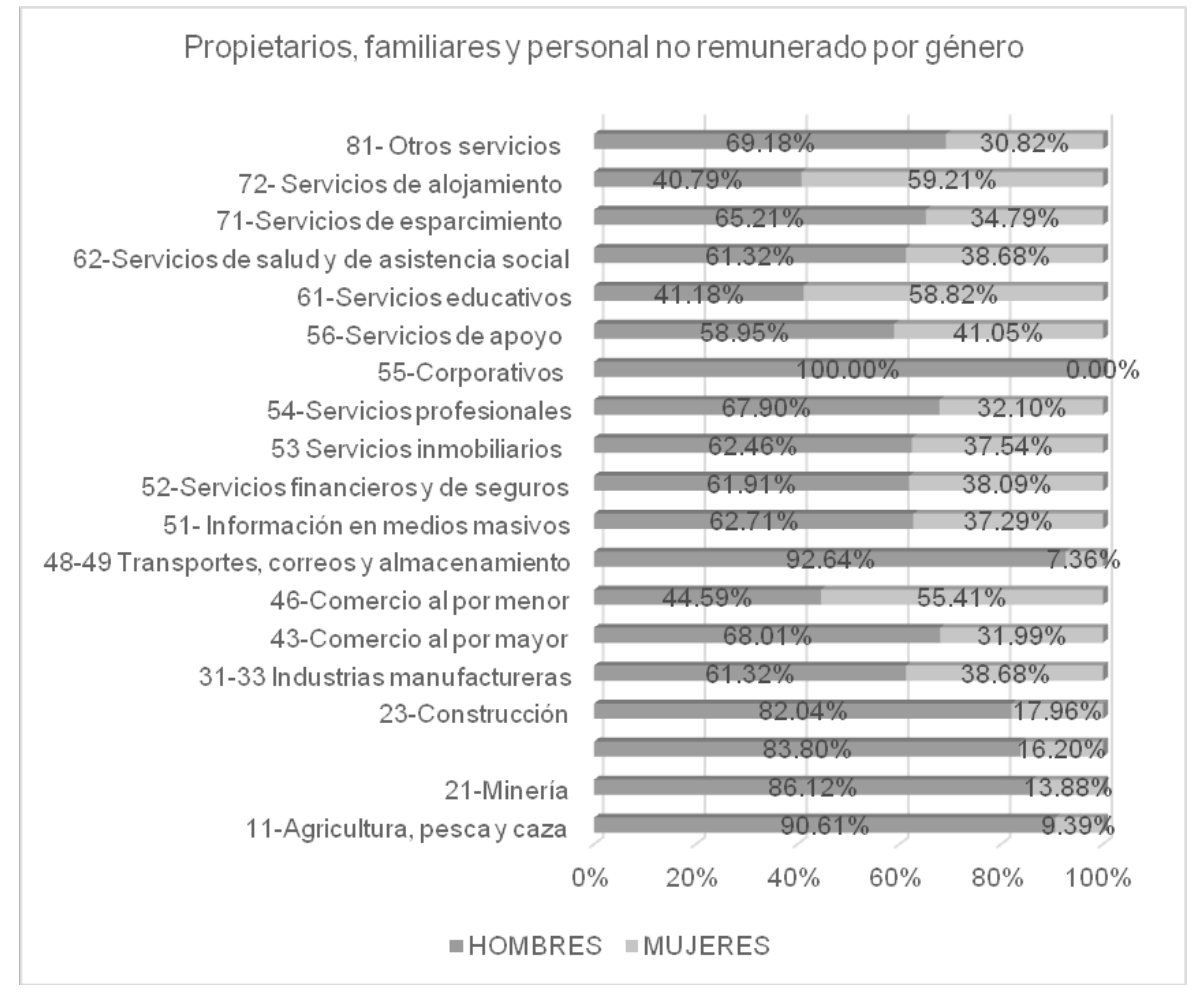

Fuente: Elaboración propia con base en los datos obtenido en el Censo Económico 2009

Horas de trabajo y la familia

Las horas de trabajo se pueden definir como un insumo importante para la producción de bienes y servicios de cualquier organización [Volpentesta, 2002]; en el caso de la empresa familiar las horas de trabajo se convierten en un insumo sin un costo financiero pero de alto valor agregado. En los datos presentados a continuación se hace una clasificación por concentración de propietarios, familiares y otros trabajadores no remunerados de concentración 
entre las horas de trabajo de los trabajadores remunerados y los propietarios, familiares y otros trabajadores no remunerados, y los sectores de actividad productiva y se encontró lo siguiente:

Los Sectores 46 (comercio al por menor), el 62 (servicios de salud y de asistencia social), el 72 (servicios de alojamiento temporal y de preparación de alimentos y bebidas), y el 81 (otros servicios excepto actividades gubernamentales) aportan más del 40\%. El sector 46 y 81 manifiesta tener más del 58\% del total de las horas de trabajo, porcentaje que establece el nivel de importancia de la familia en la empresa. (Ver Gráfica 6)

Gráfica 6 Concentración de propietarios, familiares y otros trabajadores no remunerados de concentración de horas de trabajo de trabajadores remunerados y propietarios, familiares y otros trabajadores no remunerados

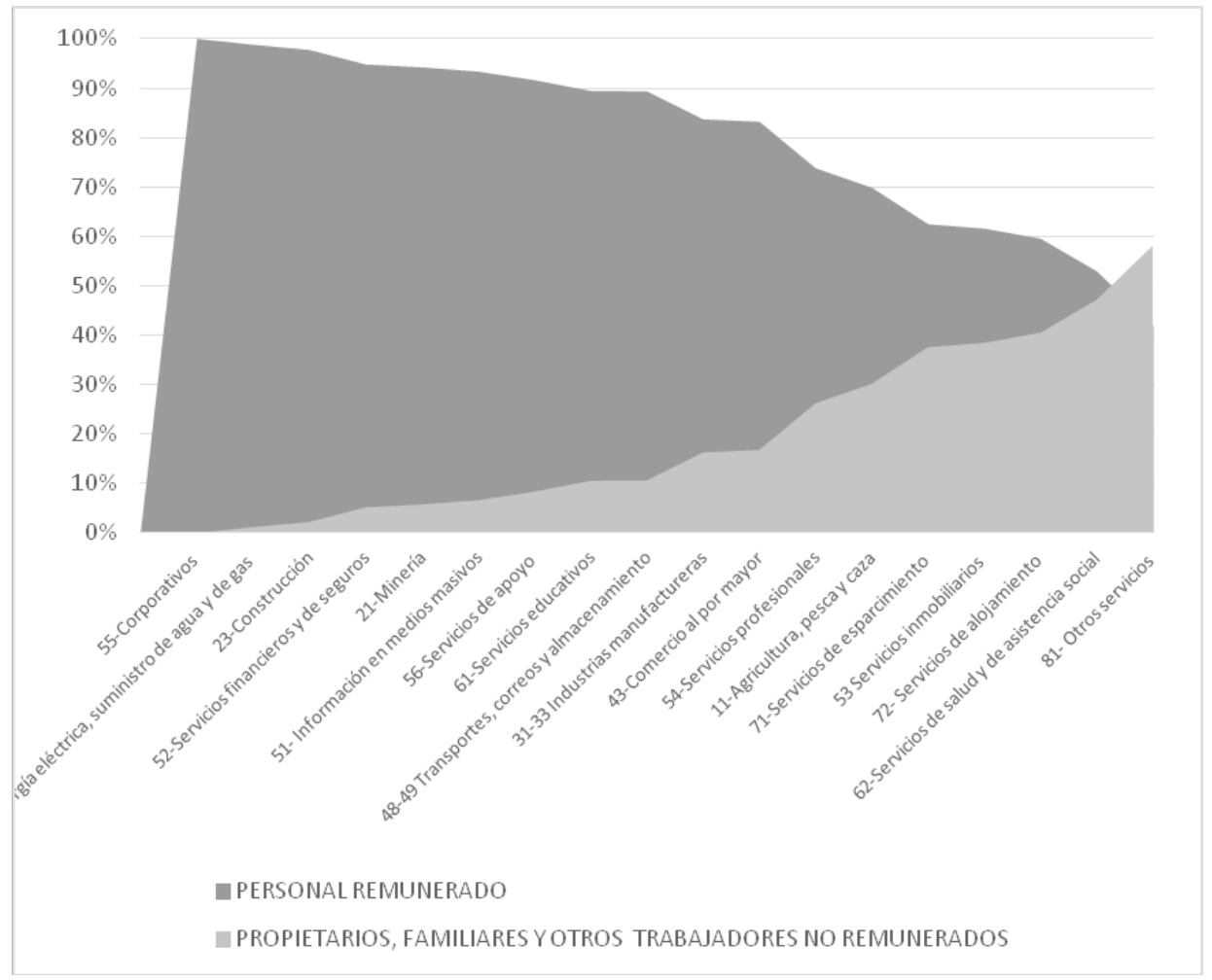

Fuente: Elaboración propia con base en los datos obtenido en el Censo Económico 2009

\section{Reflexiones}

El presente estudio de caso ayudó a identificar de forma general, el campo de estudio de la empresa familiar en México, encontró algunas estructuras sobre 
su comportamiento, por sector, por región y por tamaño. Su importancia radica en poder tener un panorama amplio y acercado a la realidad mexicana de este tipo de organizaciones; identificando algunos patrones.

- El concepto de empresa familiar es ambiguo y no nos permite tener una idea clara, sólo algunas características; tal es el ejemplo de aquellas grandes empresas que en proporción de la concentración de propietarios, familiares y otros trabajadores no remunerados es menor, y sus integrantes sólo dirigen; y por esto no son menos familiares que aquellas donde la familia es el sustento en la operatividad de la empresa, donde cada integrante aporta su conocimiento y capital de trabajo, como las horas de trabajo; cuyos insumos son importantes para la empresa que agregan valor agregado; es decir, ¿qué tan familiar es una empresa? O ¿qué define y caracteriza a la empresa familiar? El punto de reflexión alude a que, por ejemplo, aquellas empresas grandes en donde el dueño y los hijos sólo se encargan de la dirección estratégica o en aquellas que los roles de la familia se funden completamente con la dinámica de la empresa, operan, dirigen, planean y organizan; tal es el caso de TELEVISA o BIMBO, por mencionar algunas, o las micro empresas donde el papá, la mamá y los hijos tiene una obligación identificada para dar vida a la empresa. Esto en relación con los datos se menciona porque, al menos con esta base de datos, se puede decir que la mayoría de las empresas son familiares.

- En cuanto al comportamiento identificado entre la zona noroeste y noreste que tienen una menor concentración de familia, puede deberse al nivel de industrialización y de desarrollo económico y social, variables no estudiadas en el presente caso, pero sería importante tener un acercamiento a este tipo de estructuras reconocidas. Otro punto importante es poder dar explicación a que en cuanto al mapeo de la empresa familiar por regiones la concentración va de menor a mayor de norte a sur.

El género aporta otros elementos de reflexión de la empresa familiar, por ejemplo en sectores donde las mujeres tienen una mayor presencia, donde ellas han roto estereotipos y roles específicos de la cultura mexicana. La mujer en la empresa familiar resulta un punto importante sobre la agenda de investigación de la empresa familiar; así como, su inserción paulatina y creciente en la actividad económica como fundadoras, dirigentes y sucesoras en una sociedad machista [INMUJERES, 2007].

\section{Referencias bibliográficas}

Aguilar B.I. (2007), Frontera norte de México: agenda de desarrollo e integración económica. Reflexiones sobre el Noreste de México-Texas, „Revista Mexicana de Política Exterior”, (81), pp. 125-155.

Balli M.B. (2003), Negocios de familia, Bogotá, Colombia: Disonex Fonolibro. 
Barbeito R.S., Nieto de Almeida A., Pereira C D. (2011), El efecto del género en la empresa familiar, „Boletín Económico de Información Comercial Española” Retrieved from http://www.revistasice.com/CachePDF/BICE_3022_45-54__8935A1197C6463419 AB4DDF14B2FC310.pdf.

Belausteguigoitia R., Imanol (2010). Empresas familiares: su dinámica, equilibrio y consolidación, México: McGraw-Hill Interamericana.

De la Rosa A.A., Lozano C.O. (2011), Los grupos de MiPYME relativamente homogéneos como estrategia metodológica para el estudio del campo de la MIPYME, In Metodología y Estudio de la empresa familiar, pp. 105-150, México: Grupo Hess.

De la Rosa A.A. Lozano C.O.,Ramirez S.J.C. (2009), Organización, Empresa y Familia: de la Empresa Familiar a la Organización Familiar. Gestión y Estrategia, Julio/ diciembre(36), 17-36.

De la Rosa Alburquerque Ayuzabeth (2004)"Hacia la emergencia de un objeto de estudio: la micro, pequeña y mediana organización” Revista Iztapalapa No.56, año 25. México Universidad Autónoma Metropolitana Unidad Iztapalapa pp129-174.

Díaz, G. H., \& Mayett, M. Y. (2011). Diagnóstico, gestión y evolución de las empresas familiares en Tehuacán. Presented at the XVI Congreso Internacional de Contaduría, Administración e Infomática, México DF. Retrieved from http://congreso.investiga. fca.unam.mx/docs/anteriores/xvi/docs/15C.pdf

Gitman, Lawrence J y Carl McDaniel (2007) El futuro de los negocios. Traducción Pilar Mascaró Sacristán. 5a Edición. México, D. F. Editorial Thomson.

INMUJERES. (2007). El impacto de los estereotipos y los roles de género en México. INMUJERES. Retrieved from http://cedoc.inmujeres.gob.mx/documentos_ download/100893.pdf

Mendoza, C. J. E. (2006). Ingresos, integración económica y empleo en las ciudades fronterizas. Economía Mexicana. Nueva Época, XV(1), 31-65.

Mintzberg, Henry. (2002). La estructuración de las organizaciones (Séptima.). Barcelona, España: Ariel.

Ramirez, S. J. C. (2008). Relaciones de Poder, Relaciones Familiares y Empresa: El Caso de una Microorganización del Sector Textil (Maestría). Universidad Autónoma Metropolitana, México, DF.

Ruíz Durán Clemente (1995) Economía de la pequeña empresa: hacia una economía de redes como alternativa empresarial para el desarrollo. $1^{\text {a }}$ Edición. México. Editorial Planeta Mexicana.

Volpentesta, J. R. (2002). Análisis y Gestión de la productividad (1ra Edición.). Buenos Aires, Argentina: Osmar Buyatti.

Base de datos:

Censo Económico 2009. INEGI. 